\title{
Article \\ Experimental Comparison of Field and Accelerated Random Vertical Vibration Levels of Stacked Packages for Small Parcel Delivery Shipments
}

\author{
Bence Molnár and Péter Böröcz *(D) \\ Department of Logistics and Forwarding, Széchenyi István University, Egyetem tér 1, 9026 Győr, Hungary; \\ molnar.bence@sze.hu \\ * Correspondence: boroczp@sze.hu; Tel.: +36-70-335-22-60
}

check for

updates

Citation: Molnár, B.; Böröcz, P. Experimental Comparison of Field and Accelerated Random Vertical Vibration Levels of Stacked Packages for Small Parcel Delivery Shipments. Appl. Sci. 2021, 11, 2927. https:// doi.org/10.3390/app11072927

Received: 22 February 2021

Accepted: 22 March 2021

Published: 25 March 2021

Publisher's Note: MDPI stays neutral with regard to jurisdictional claims in published maps and institutional affiliations.

Copyright: (C) 2021 by the authors. Licensee MDPI, Basel, Switzerland. This article is an open access article distributed under the terms and conditions of the Creative Commons Attribution (CC BY) license (https:// creativecommons.org/licenses/by/ $4.0 /)$.
Abstract: In the last decade, there has been a significant increase in parcel delivery shipments all over the world due to online stores and consumer demand to receive the products in a shorter period of time. It is especially true when situations like COVID-19 limits personal purchases in shopping malls as well as grocery and pharmaceutical stores. This often means that courier operators try to deliver packages utilizing vehicles with racks or shelves, which during the COVID-19 epidemic are not there anymore. This study measured the vertical vibration levels that occur in stacked parcels during express delivery versus the simulation technique in the laboratory. The goal of this paper was to measure and compare the vibration levels between laboratory practice and field transportation. For the measurement a three-layer stacked unit was built to observe the vibration levels on different road conditions in a parcel delivery vehicle and ASTM vibration profile (ASTM International). Then the measured acceleration-time data were analyzed in terms of power spectral densities (PSD) and the presented statistical data provided an understanding of the variability of intensity in different levels in stacked unit. The results showed that the vibration level increases in the stacked load upwards and with worse road conditions, but even in the worst case it did not reach those vibration levels that the laboratory test showed. Moreover, the layers of the stacked unit are in out-of-phase motion in the field, while the stacked unit in the vibration simulation usually is in-phase motion. Results indicate that the proposed vibration simulation does not correlate well with typical field vibration. This is fundamentally true as during a forced vibration created by a single-axis shaker, do not account for additional inputs occurring simultaneously creating an off-balance to the loads and as a result are less severe than simulated conditions. These findings are limited to single axis vibration simulation and unsecured loads.

Keywords: vibration; power spectral density (PSD); stacked packages; parcel delivery

\section{Introduction}

The global courier, express and parcel market has grown steadily over the past decade and the net income of these services doubled in this time [1]. In this service, the first and last step of distribution occurs by small vehicles, when the operators collect or deliver the parcels. This paper focuses on this part of the transportation. All over the world, the volume of travel for parcels has exponentially increased, so better testing is needed. Companies like Amazon (www.amazon.com, accessed on 15 January 2021) and carriers like UPS (www.ups.com, accessed on 15 January 2021) are now the global leaders in providing online sales for customers and providing delivery of parcels. Amazon this year started to deliver packages, on the way to become the largest parcel delivery company.

This mode of express delivery brought new circumstances during transportation. The huge amount of goods causes a very intensive flow in the distribution channel by collecting and delivering single and various products day by day. This often means that the operators try to use the most of the vehicle capacity; thereby many identical or different kinds of 
parcels are stacked on top of each other. This situation is complicated further by the fact that the fixation accessories such as fixing straps or bands are virtually non-existent in this transportation mode. This is a common practice in Europe, but on some continents, this is different because the operators use vehicles equipped with shelves. Figure 1a,b shows some examples for parcel shipments during daily courier service.
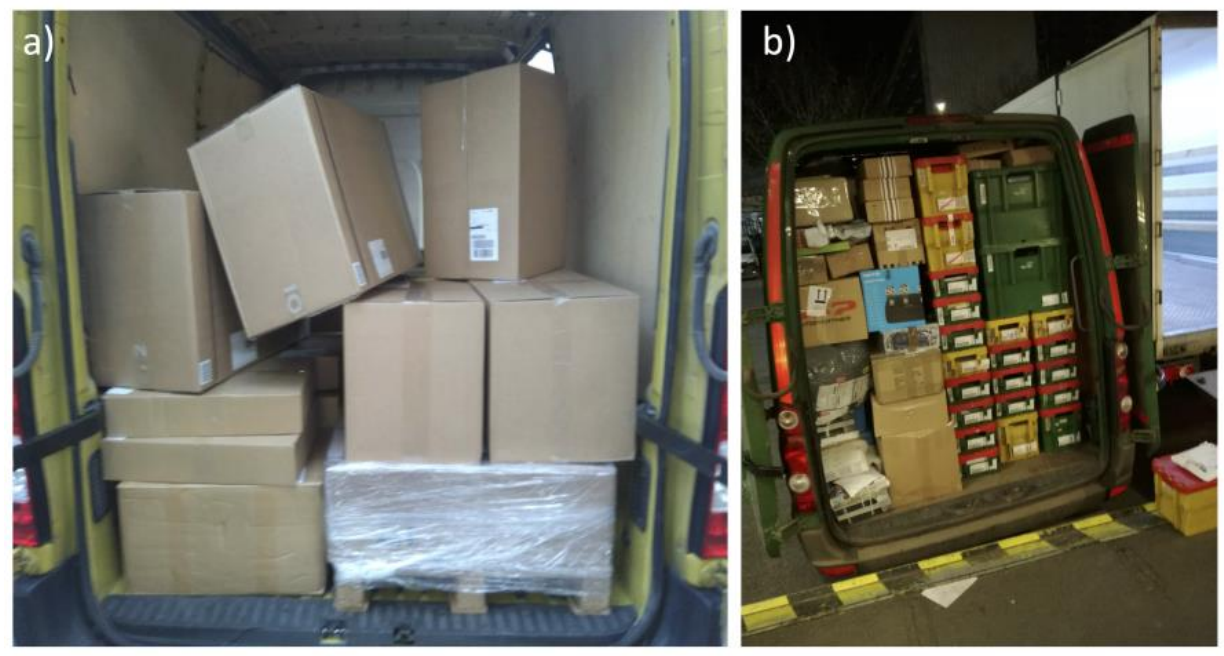

Figure 1. Stacked parcels during daily express delivery service, (a) mid-sized packages (b) small packages.

The shipments for courier parcel packages usually consist of small size, lightweight and time sensitive products. The vibration environment of these stacked shipments produces unique physical events due to free movement spaces around them [2]. Since they are not restrained as unitized loads with stretch wrap or banding. In the vertical direction these parcels practically can move freely. This physical condition is very important to analyze in order for packaging engineers to design suitable protective packaging systems.

The vibration environment during distribution can be based on factors such as vehicle body structure, type of vehicle suspension and tires, road roughness, vehicle speed and actual payload, respectively [3]. Previous studies have measured and analyzed vibration levels for parcel delivery vehicles and CEP (Courier Express Parcel) modes of transportation [4-9]. Factors regarding the effect of payloads, suspension system and road condition to vibration levels during express in delivery vehicles have also been previously studied $[4,9,10]$. However, all this previous research measured and analyzed the vibration levels on the floor of the vehicle and none of them took into consideration that most of these parcels are transported in "unrestrained stacked configuration" and without shelving due to the intensive flow of goods and relatively limited capacity of vehicles. Furthermore, most of the previous studies did not investigate the differences and similarities between the laboratory simulation and field transportation. The vibration levels that is experienced in stacked loads (in layers) is different from the direct input citation that can be observed on the vehicle floor. Furthermore, these parcels do not act as a unitized pallet as they are not fin contact or restrained to each other.

Another aspect is that the results of study are also important, namely the generally widely used protocols for parcel shipment simulation that do not deal with a stacked unit. The only one, which separately deals with small parcel delivery shipments, is the ASTM D7386 [11]. However, this protocol also does not expose specimens for stacking in order to test the upper layers if it is made from small packages. The only way to try to simulate small parcel delivery is a so-called vibration testing under dynamic load, which means that there is a top load bag apparatus for the test specimen that tries to simulate an additional load above the packages.

The main goal of this paper was to measure the vibration levels in layers of stacked packages in parcel delivery shipments and to compare the results to the laboratory experi- 
ence and show the differences. The new results can be useful for packaging engineers to make better and precise pre-shipment testing. Furthermore, the results support the use of the vibration technique in the simulation of parcel delivery goods in a stacked way. It is important to note that existing ASTM method are not an under-test causing damage, but an over test.

\section{Experimental Design}

\subsection{Samples and Measuring Equipment}

For this study three identical small, corrugated box parcels were set up for the measurements of the stacked unit. Each sample packaging contained a Lansmont SAVER $3 \times 90$ field data recorder (Lansmont Corp., Monterey, CA, USA), which was fixed to an aluminum frame (Figure 2). Inside the package this ITEM aluminum profile frame (@item Industrietechnik $\mathrm{GmbH}$, Solingen, Germany) ensured the rigid fixation of SAVERs and the best fitting to the boxes' geometrical sizes. Thereby, a total of four SAVERs were used for the measurements, three of the SAVERs were in the sample boxes and one of the SAVERs was mounted to the van's platform directly. Table 1 describes the sample specifications and SAVER settings for this study.
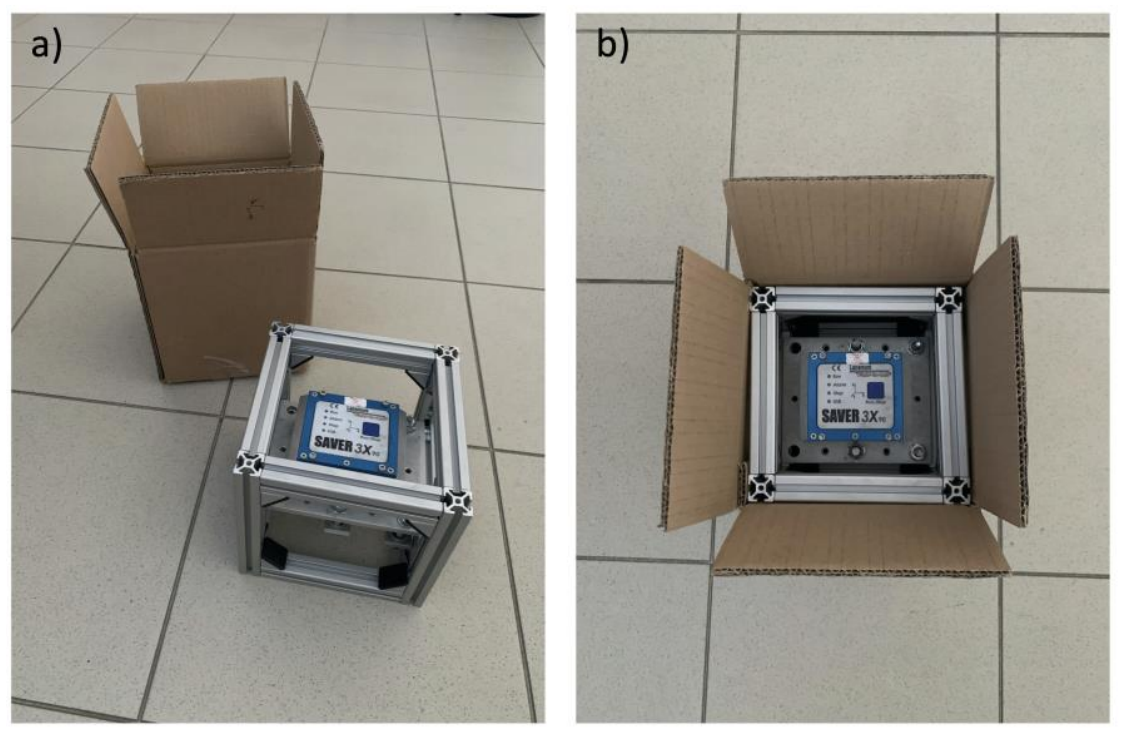

Figure 2. Applied sample packaging during the measurements, (a) the box and recording instrument (b) the instrument placed

Table 1. Specifications for SAVER settings and sample used for this study.

\begin{tabular}{cccc}
\hline \multicolumn{2}{c}{ Samples Used } & \multicolumn{2}{c}{ SAVERs } \\
\hline Corrugated board & $35 \mathrm{BC}$ & Timer triggered data & $1 \mathrm{~s}$ \\
Weight of board & $742 \mathrm{~g} / \mathrm{m}^{2}$ & Wake-up interval & $1 \mathrm{~s}$ \\
ECT & $9.0 \mathrm{kN} / \mathrm{m}$ & Recording time & $1.000 \mathrm{~s}$ \\
BST & $1685 \mathrm{kPa}$ & Sample $/ \mathrm{sec}$ & $500 \mathrm{~Hz}$ \\
Weight of box & $190 \mathrm{~g}$ & Sample size & 500 \\
Size of box $(\mathrm{w} \times \mathrm{d} \times \mathrm{h})$ & $180 \times 180 \times 195 \mathrm{~mm}$ & Frequency resolution (PSD) & $0.50 \mathrm{~Hz}$ \\
Weight of ALU frame & $1440 \mathrm{~g}$ & Anti-Aliasing frequency & $200 \mathrm{~Hz}$ \\
Entire weight & $2630 \mathrm{~g}$ & & \\
\hline
\end{tabular}

\subsection{Measurement Setup}

One measurement system was built for the field and one for the laboratory standard test in order to obtain and compare vibration levels. In the first step, the field vibration levels were measured, and for this a FIAT Ducato (2008, 2.2. Multijet, load capacity of $11 \mathrm{~m}^{3}$, distance between front and rear axis $4035 \mathrm{~mm}$, one parabolic leaf spring at rear) was 
used. The stacked unit was located above the rear axle of the van and an aluminum fence was used to prevent the stacked unit from moving in lateral and longitudinal directions. The stacked unit motion in the vertical direction was not restricted. The fourth SAVER was mounted to the platform with screws as close as possible to the stacked unit. This setup can be seen in Figure 3a.
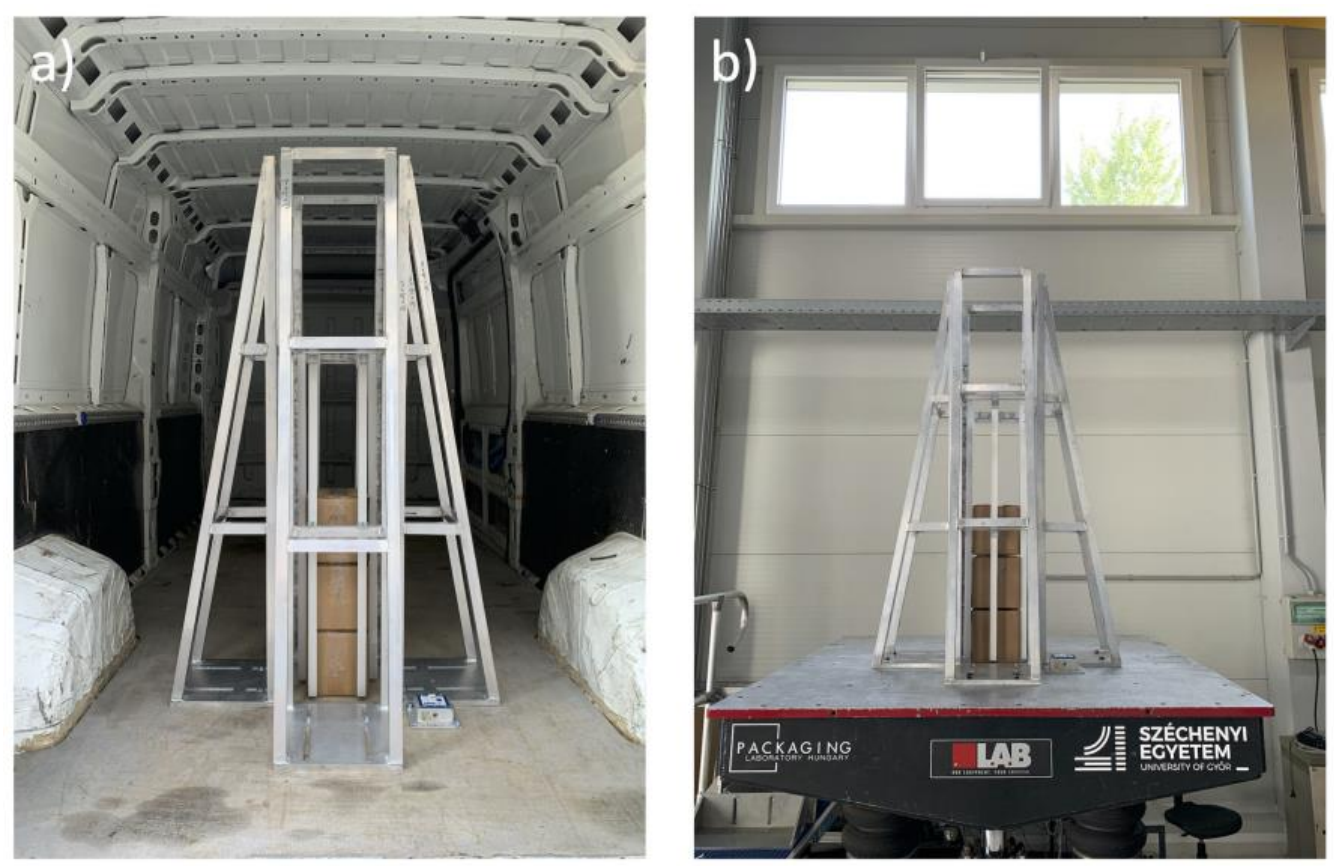

Figure 3. (a) Sample stacked unit in van and (b) in laboratory.

For the second step, the standard laboratory vibration test was performed to measure the vibration levels on the same stacked unit. For this vibration test a hydraulic vibration table (LAB HV-60) was used with the same accessories as in the field. Figure $3 b$ shows the laboratory test setup.

\subsection{Delivery Routes and Vibration Profile}

To collect the field vibration data four different route conditions were used. The measurements were performed on motorway, arterial road, country road and urban roads, respectively. The reason for choosing these categories is to compare the vibration levels between varied road conditions and to search for that vibration level which is closest to the accelerated laboratory circumstances. Each measurement was taken for an approximately 60 min period per routes. The measurements were executed at night only, so the van could maintain a relatively higher travelling speed due to less traffic. This is important due to the fact that a higher vehicle speed produces higher vibration intensity $[4,12,13]$. The routes for the measurements can be seen in Figure 4 and Table 2 contains information of distances and average vehicle speeds. The experiment was conducted from 6 to 7 August (2020) in the county of Győr-Moson-Sopron, Hungary. 
Table 2. Specifications of routes and vehicle speeds for the van used in this study.

\begin{tabular}{ccc}
\hline & Distance & Average Speed \\
\cline { 2 - 3 } & $\mathbf{( k m )}$ & $\mathbf{( k m / h )}$ \\
\hline Motorway & 120.9 & 114 \\
Arterial & 80.2 & 73 \\
Country & 51.5 & 54 \\
Urban & 38.4 & 35 \\
\hline Summary & 291 & 69.1 \\
\hline
\end{tabular}
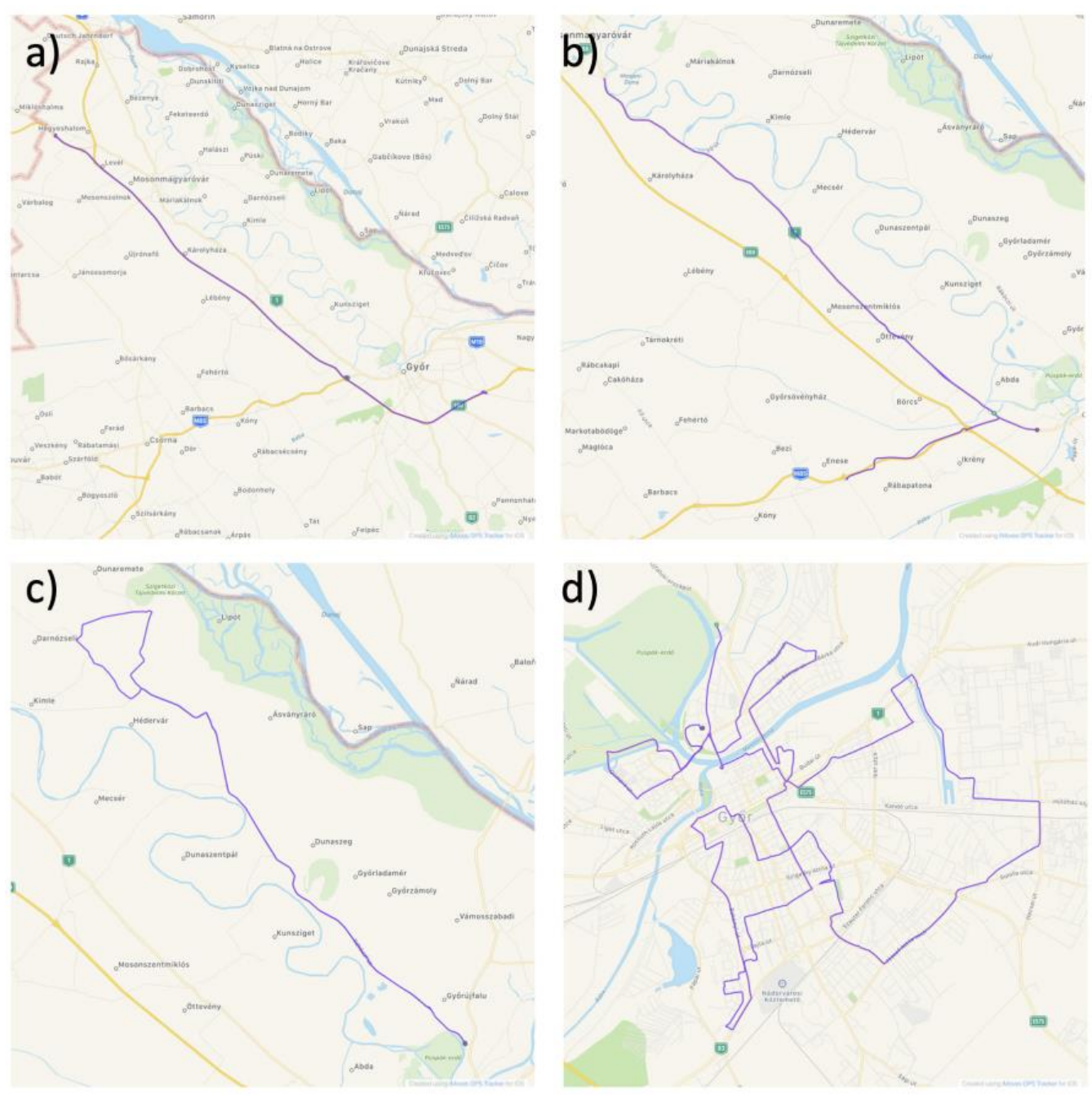

Figure 4. Measured routes (a) Motorway, (b) Arterial road (c) Country road (d) Urban road.

The laboratory measurement was performed at the Packaging Laboratory, Hungary (University of Győr). The ASTM D7386 Standard Practice for Performance Testing of Packages for Single Parcel Delivery Systems (Figure 5) was programmed for the vibration system. This testing procedure is the one generally used for packed-products for parcel delivery shipment. This PSD spectrum simulates small vehicle vibration in the test procedure. The frequency range and overall $\mathrm{G}_{\mathrm{rms}}$ of this profile is $1-200 \mathrm{~Hz}$ and 0.46 . 


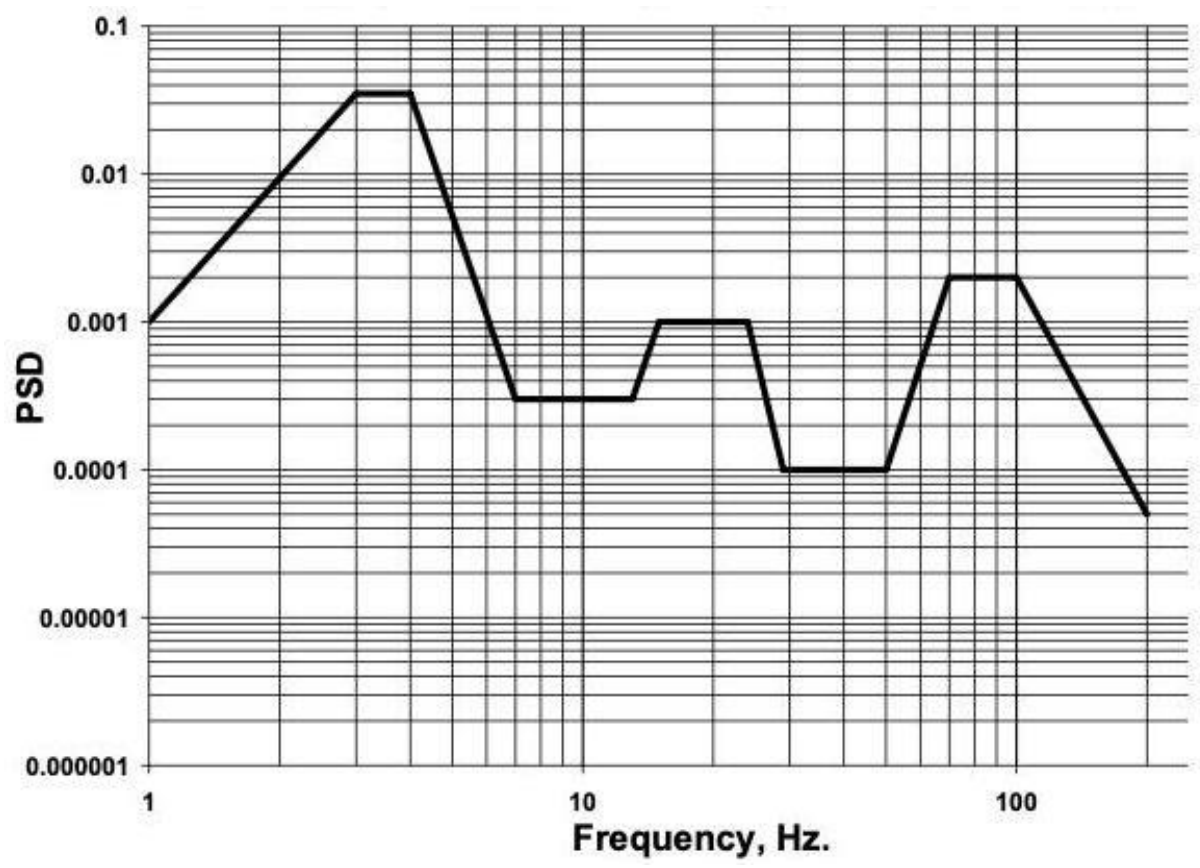

Figure 5. ASTM D7386-Standard Practice for Performance Testing of Packages for Single Parcel Delivery Systems PSD spectrum.

\subsection{Statistical Methods for Acceleration Peaks and RMS ( $g$ )}

Statistical analyses of the collected vibration events were performed with MATLAB R2014a (MathWorks Inc., Natick, MA, USA). First the recorded vertical acceleration peaks and calculated RMS (root mean square) values were grouped by road conditions and layer levels and were analyzed using one-way ANOVA (analysis of variance) to compare the differences among groups with the presentation of bar graphs. In this step a post-hoc multiple comparison range test was also used (Tukey's). The significance level for this analysis was determined at $5 \%(p<0.05)$. Next, the measured vertical acceleration peak (g, peak) and RMS (g) were analyzed using empirical cumulative distribution functions (CDF). It represents the percentage of events below a specific value, which helps to more thoroughly understand the vibration behaviors of a given layer of stacked unit. In addition, the statistical distributions of the measured acceleration peaks (g, peak) and root mean square (RMS, g) were calculated for the recorded data in layers to construct the kurtosis (K) and skewness (S) of the probability distribution functions (PDF) for these events using a $95 \%$ confidence interval (CI). Kurtosis and skewness show the asymmetry and peakedness deviation of data from the Gaussian normal distribution [14,15]. Kurtosis can be computed as Equation (1) shows.

$$
\sum_{i=1}^{N}\left\{\frac{\chi_{i}-\mu}{\sigma}\right\}^{4}
$$

\subsection{Data Analyzes of Power Spectral Density (PSD)}

PSDs were prepared from the measured vibration data using Fast Fourier Transformation (FFT) of Xware software and MATLAB R2014a (MathWorks Inc, Natick, MA, USA). The values of power density (PD) levels are presented between 1-200 Hz. This frequency range represents those vibration events, which have enough intensity to influence the integrity of product-package system used in general industry (Figure 5). Furthermore, this frequency range contains those vibration responses that can be observed in small vehicle delivery from the vehicle body structure, suspension system, tires, etc. Due to less traffic and the lack of stopovers in this study, the recorded data was not additionally filtered to remove any undesirable events. The PSDs are presented for each layer of the stacked unit and for the platform of the van on each road condition and in the laboratory measurement 
as well. For easier comparability, the spectra of the ASTM D7386 is also shown in the figures. Additionally, the overall $\mathrm{G}_{\mathrm{rms}}$ were calculated from the spectra in each case and layer between 1-200 Hz.

The average power density (Equation (1)) within a narrow band of frequencies $(B W)$ of a given spectrum can be determined by $G_{r m s}$ values based on the number of samples of a given bandwidth. In this way $G_{r m s}$ is determined by the root mean square value of the acceleration in G's in the given bandwidth of frequency, based on the number of (n) samples.

$$
P D=\frac{1}{B W} \sum_{i=1}^{n}\left(R M S G_{i}^{2}\right) / n
$$

\section{Results and Discussions}

\subsection{Results of Acceleration Peaks and RMS $(\mathrm{g})$}

Figure 6 shows the bar graphs of recorded peak acceleration and RMS acceleration values for all layers and floor and presents the data as a function of road conditions. As it was expected the worst road conditions (urban road) produced the highest values. Obviously, relatively bad road conditions are the reason for this phenomenon. Despite the lower travelling speed, the highest values occurred on urban roads where many road defects and unrenovated roads can be found. Based on layers the highest values were in the 3rd level followed by 2nd, and the least in 1st level, respectively. In the field, the floors' acceleration values were always the lowest, and the values increased upwards in the layers. However, in laboratory test the platform values were the highest and acceleration values were generally lower in the top three levels, of course increasing upward level by level. The reason of the later phenomenon is that the test standards' vibration profile applies timecompression, which artificially amplifies the vibration magnitude at laboratory test. At this vibration level the cushioning features of the corrugated box structure can prevails better.

a)

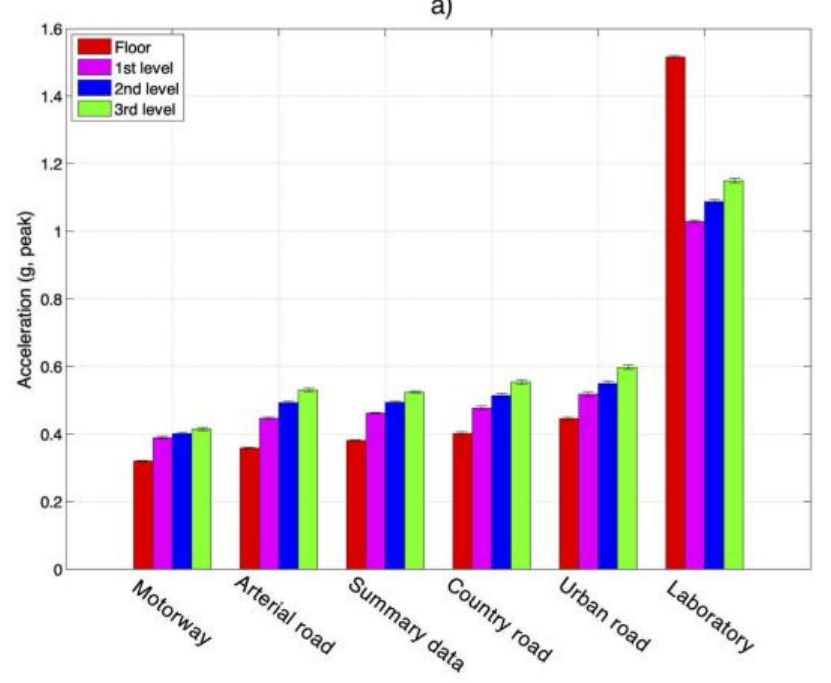

b)

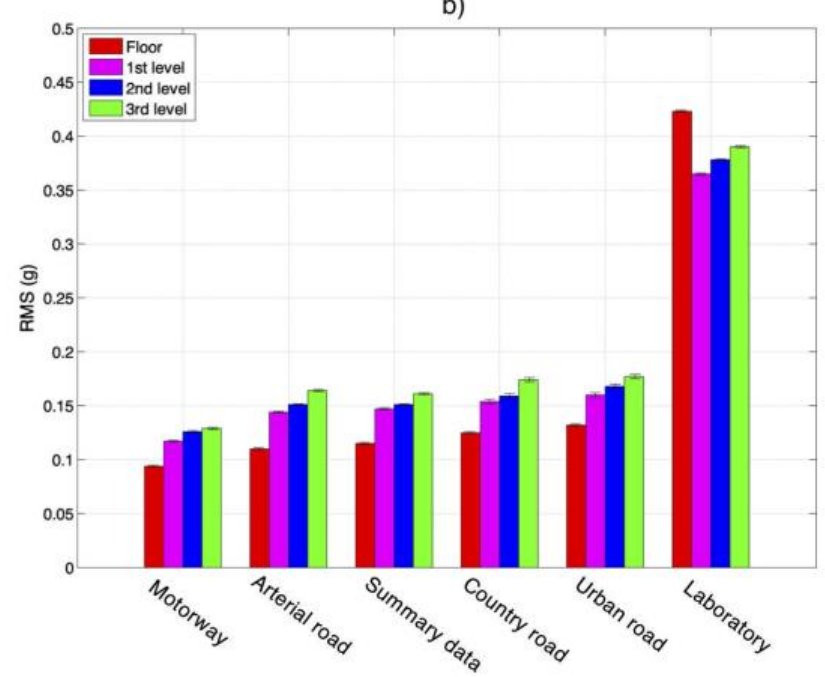

Figure 6. (a) Peak acceleration and (b) RMS acceleration values of the stacked unit and floor on different road conditions.

There were no statistically significant differences at acceleration peaks between the 1st and 2nd level for the motorway; between arterial road and country road at acceleration peaks and RMS acceleration values for the 2nd level; between the 2 nd and 3rd level of RMS acceleration for the motorway $(p<0.05)$. In the laboratory, the acceleration values on the platform were 1.31-1.48 times higher than those measured in the upper levels of the stacked unit. However, in the field measurements, the acceleration values on the floor were 0.72-0.82 times lower than in the higher levels. This can be seen in the summary data. In terms of summary data for stacked unit levels the acceleration values were 1.21, 1.29 
and 1.37 times higher (1st, 2nd and 3rd level) in comparison to the floor. The acceleration values also showed an increasing tendency in the unit rows in laboratory test compared to each other's, but not to the platform of the vibration system.

\subsection{Empirical CDFs for Acceleration Peaks and RMS (g) Data}

Figures 7 and 8 show the CDFs (Cumulative Distribution Function) of the acceleration peak and RMS data, it represents the percentage of the events that are below a given acceleration peak value or RMS level. The CDFs were calculated and plotted from data of each road condition, laboratory test and stacked levels, respectively.
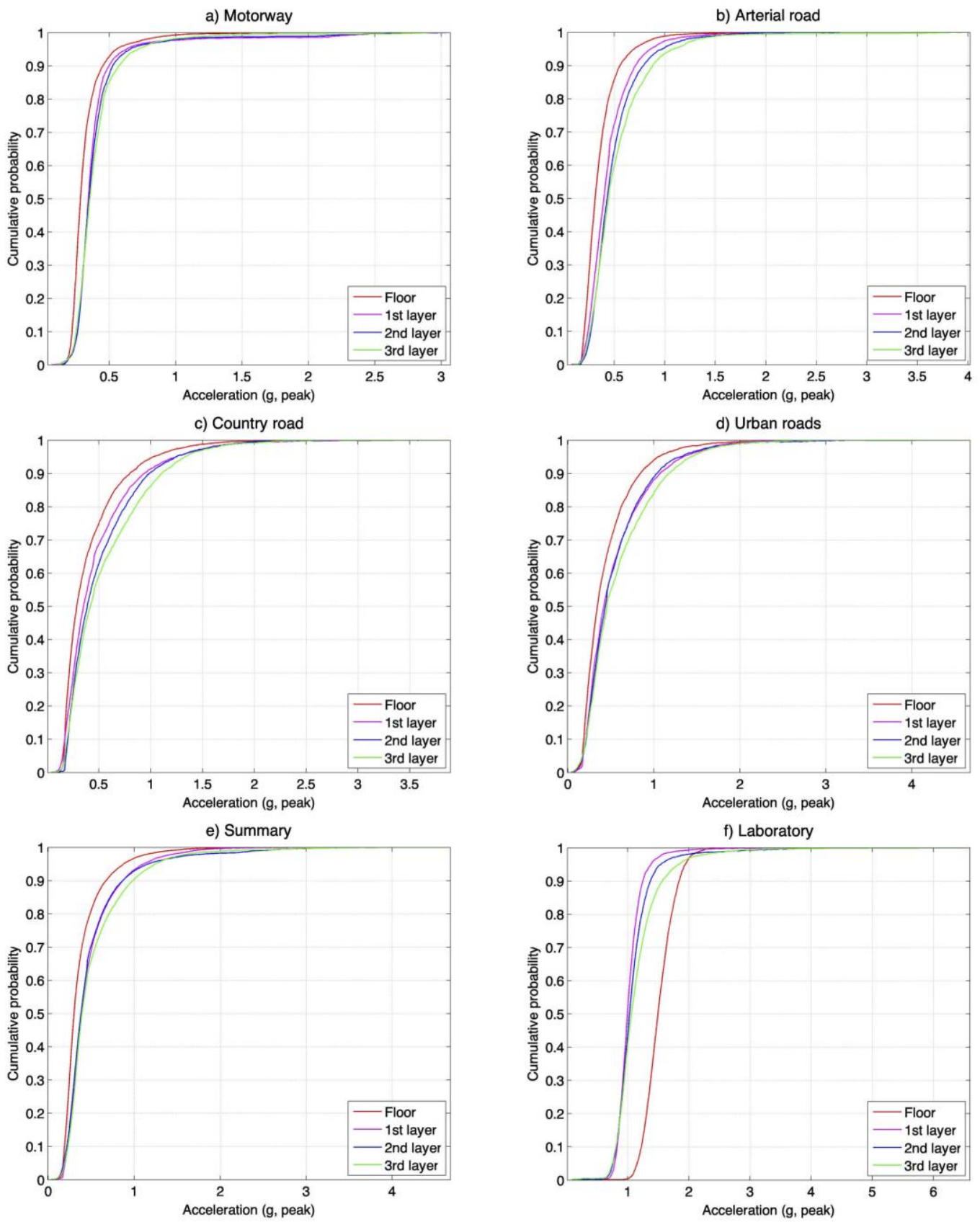

Figure 7. Empirical CDFs of peak acceleration values, (a) Motorway, (b) Arterial road, (c) Country road, (d) Urban roads, (e) Summary, (f) Laboratory. 

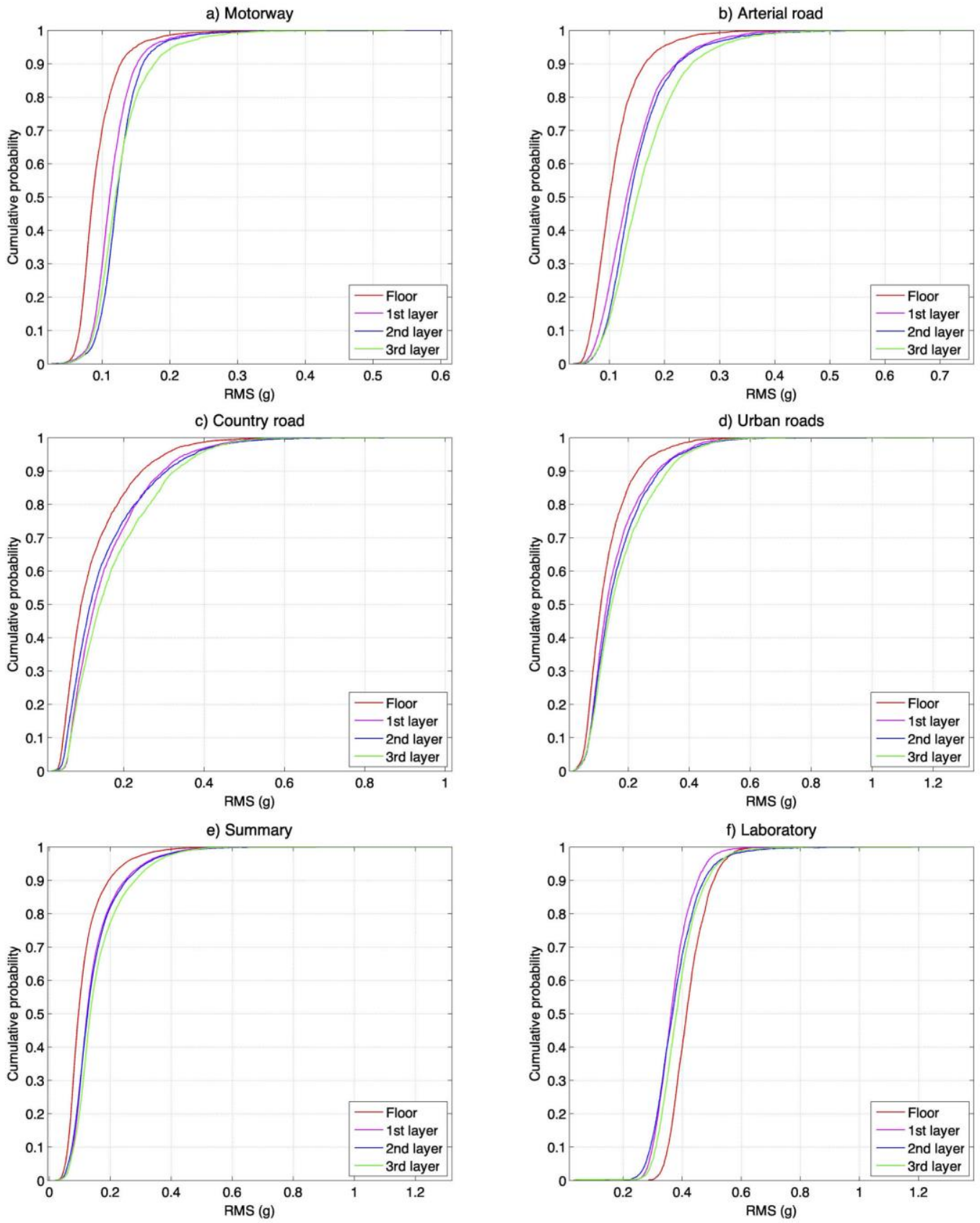

Figure 8. Empirical CDFs of RMS (g) values, (a) Motorway, (b) Arterial road, (c) Country road, (d) Urban roads, (e) Summary, (f) Laboratory.

The acceleration data (Figure 7) shows that the highest acceleration values were recorded on urban roads in the field, and on the van's floor $90 \%$ of the total events on the floor were below $0.85 \mathrm{~g}$ and in the 3 rd level were below $1.18 \mathrm{~g}$, which are about 2 times higher than the measured values on the motorway in layers 1-3, and 1.8 times higher than it was on the floor. In the case of the laboratory test the data shows that $50 \%$ and $90 \%$ of the values on the floor were below $1.49 \mathrm{~g}$ and $1.84 \mathrm{~g}$, which is 2.2 and 4.35 times higher than in the case of urban roads, but these significant differences do not appear in stacked layers data. Here these values were 1.2 times (for 1st layer), 1.28 times (2nd layer), and 1.33 times (3rd layer) higher. Comparing the laboratory test and summary data for field, at $90 \%$ occurrence, it can be seen that laboratory events were 2.73 times higher on the floor, $1.43,1.58$, and 1.59 times higher upwards in the layers. It is a very interesting observation that in the laboratory test the floor curve crossed the 3rd and 2nd layer's curve at the point 
of $96 \%$ and $98 \%$, which can mean that some vibration events generated by the vibration controller produce higher acceleration events on stacked unit layers. This phenomenon can also be observed in the CDFs curve for RMS (g) data.

In terms of RMS data (Figure 8) it can be clearly visible that the functions of stacked layers almost coincide below $50 \%$ probability. The highest RMS value at this point was calculated in the 3rd layer on urban roads, it was 1.25 RMS (g), and on the floor the highest was 1.11 RMS (g). Comparing these values with the data obtained from the motorway these were approximately 1.9 times higher in each layer at the $90 \%$ occurrence level. As laboratory test results show $90 \%$ of the RMS values were below 0.51 RMS (g) on the floor, and $0.45,0.47$ and 0.49 RMS (g) from the 1st to 3rd layers and these values were 2.6, 1.8, 1.9 , and 1.7 times higher than the values of the field summary data from floor to 3rd layer, where those were $0.20,0.25,0.26$ and 0.28 RMS (g), respectively. Appendices A and B contains specifications for CDSs of each measurement that were conducted for this study.

\subsection{Power Spectral Density Plots (PSDs)}

The measured data were analyzed to determine the PD levels of vibration in field and laboratory measurements. Figure 8 shows the developed PSDs of each layer of the stacked unit and floor and supplemented with the spectrum of ASTM D7386. It can be seen that the measured PD levels are almost the same in each layer between 1-8 Hz in the case of field measurements, and 1-6 Hz in the laboratory. Except for the laboratory, the PD levels in that frequency range did not reach the level of the test spectrum, but they increased in the function of the road condition. The observed vibration levels on the floor decreased from about $18 \mathrm{~Hz}$ on all road conditions, but in the case of country and urban roads between $29-48 \mathrm{~Hz}$ this vibration level almost coincides with the test spectrum of ASTM D7386. It is worth noting that each stacked layer shows higher PD levels in a difference frequency range than in the field measurement data, where they are higher than the other layers and the test spectrum; the highest PD levels were recorded on the 3rd layer between 9-14 Hz and $19-33 \mathrm{~Hz}$, on the 2nd layer between $16-19 \mathrm{~Hz}$, and on the 1st layer between $35-51 \mathrm{~Hz}$. All stacked layers rapidly decreased above $50 \mathrm{~Hz}$, and they were below the floor vibration intensity above $55-60 \mathrm{~Hz}$. These phenomena can also be observed in the laboratory, with the difference that the frequency ranges of the phenomena are different. In this case the highest PD levels are recorded on the 3rd layer between $8-22 \mathrm{~Hz}$, on the 2nd layer between $22-33 \mathrm{~Hz}$, and on the 1st layer between $33-56 \mathrm{~Hz}$.

At this point it is so important to emphasize that the vibration simulation protocol generally uses time-compression method by increasing the vibration magnitude during simulation [16,17], presumably ASTM protocol also applies this. However, it can be clearly seen in Figure 9 that this artificial gain is not evenly distributed over the entire frequency range studied. The PD levels of the layers in the stacked unit reached or exceeded the ASTM spectrum levels in the frequency range of $7-50 \mathrm{~Hz}$, so here the artificial amplification of the test spectrum cannot be interpreted. In the 1-6 Hz lower frequency range the field PD levels, even in the higher stacking level, are much lower than the protocol values. Over $50 \mathrm{~Hz}$ the response vibration of the unit rows is also lower than the test spectrum. So, in these two frequency regions, the assumed time-compression can be determined even for the top level of a stacked unit without fixation. Another observation is that the motion of the stacked unit deviates the motion of both vibration table and the delivery vehicle between $8-50 \mathrm{~Hz}$. 

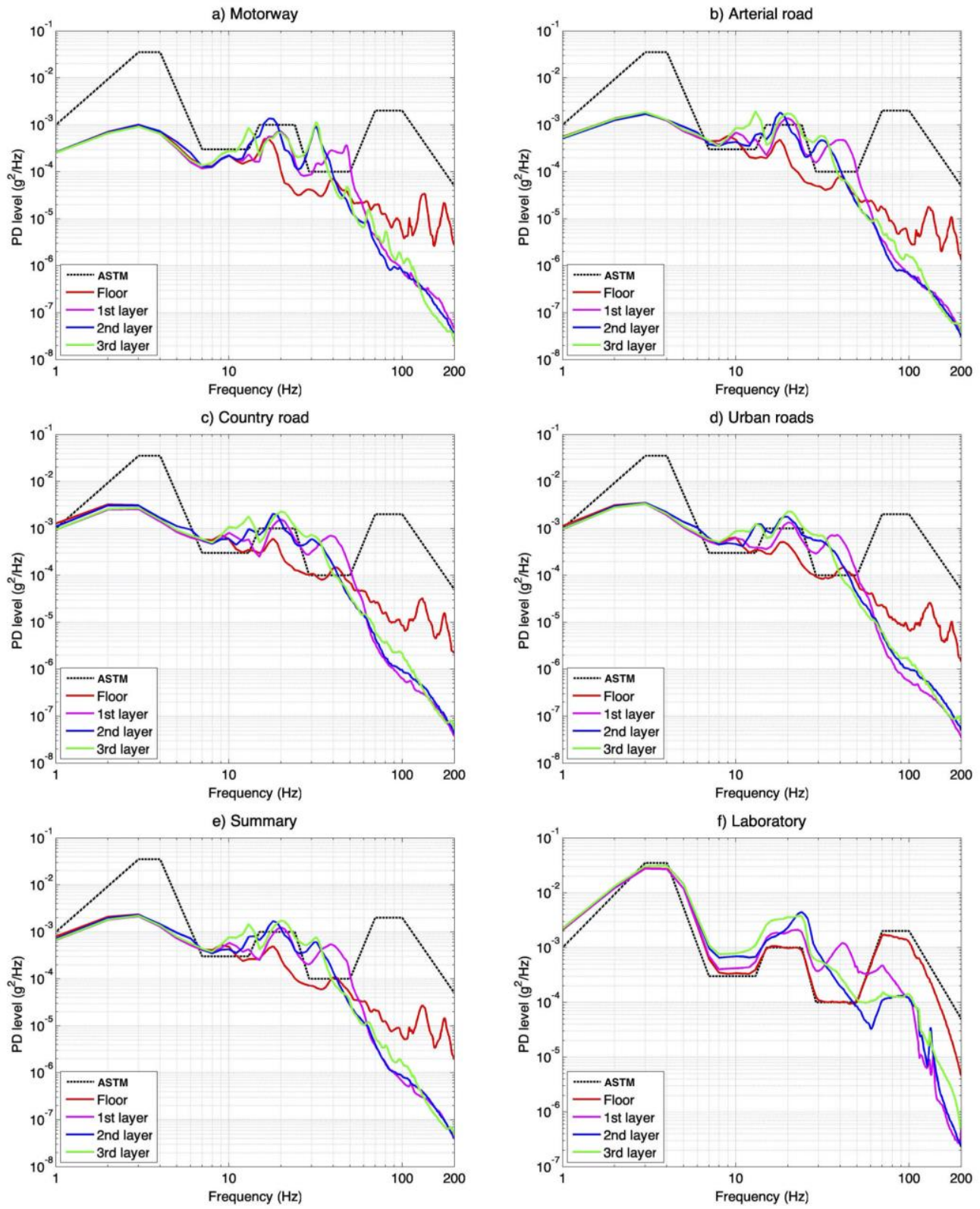

Figure 9. PSD plots of various layers in a stacked unit, on the floor and ASTM D7386 spectrum at, (a) Motorway, (b) Arterial road, (c) Country road, (d) Urban roads, (e) Summary, (f) Laboratory.

It should be emphasized here that the ASTM vibration test protocol is based on field measurements that were conducted in the US with parcel delivery vehicles [4] and are used as worldwide protocol in the packaging testing practice (as practically the only one for parcel package delivery vibration testing), but some former studies showed that the vehicles that used in outside of US produce slightly different PSD shape in the 1-200 Hz frequency range [4]. Furthermore, the PSD plot obtained for this study shows a little bit lower intensity than it was observed in former published research [4]. The reason can be that this paper used only one specific vehicle to observe the vibration event in the field focusing on the vibration levels in unit load, until the previous study applied several different parcel delivery vehicles to get information about the vibration levels.

The overall $\mathrm{G}_{\mathrm{rms}}$ values were calculated for each layer of the stacked unit and also for the floor. These data are presented in Table 3. In the analyzed frequency range $(1-200 \mathrm{~Hz})$ the values increased upward except in the laboratory where this tendency was true for the stacked unit but in this case the highest $\mathrm{G}_{\mathrm{rms}}$ value was on the floor. From the data it can 
be determined that there is no significant difference between the overall $G_{r m s}$ values of country and urban roads.

Table 3. Overall $\mathrm{G}_{\mathrm{rms}}$ of various layers and the source of data $(1-200 \mathrm{~Hz})$.

\begin{tabular}{ccccc}
\hline Location & Floor & 1st Layer & 2nd Layer & 3rd Layer \\
\hline Motorway & 0.099 & 0.122 & 0.131 & 0.135 \\
Arterial road & 0.119 & 0.164 & 0.156 & 0.177 \\
Summary & 0.136 & 0.166 & 0.170 & 0.181 \\
Country road & 0.151 & 0.187 & 0.187 & 0.203 \\
Urban road & 0.154 & 0.187 & 0.197 & 0.204 \\
Laboratory & 0.427 & 0.376 & 0.387 & 0.397 \\
\hline
\end{tabular}

\subsection{Statistical Characteristics of Acceleration Peaks and RMS $(g)$}

Figure 10 shows the calculated probability density functions of the RMS $(\mathrm{g})$ data from field measurement and laboratory measurement and each figure shows the calculated kurtosis and skewness values. Side-by-side diagrams help compare data from laboratory tests and field measurements. In the case of random vibration test, the controller operates with Gaussian distribution, but by specifying the kurtosis distribution the suitable controllers can perform the random vibration test in a more realistic way.

In the case of the calculated distribution for the RMS (g), the kurtosis values in each case were positively peaked. In both the field and laboratory cases the highest kurtosis values were in the 3rd layer of the stacked unit with a value of 35.85 for field, and a value of 6.54 for laboratory (Appendix C). The increasing tendency for kurtosis value upward in the stacked unit can be seen in Figure 10. These phenomena are in line with the previous papers' results in which the non-Gaussian nature of random vibrations on delivery vehicles were investigated [18-21]. The skewness values in each case were positive and between the values of $1.62-2.63$.

\subsection{Limitations for Practice}

1. The applied PSD method for the data of this study uses the Fast Fourier Transformation (FFT) process, so PSD plots show averaged intensity over the observed frequency range of interest along the data collected, thereby the plots do not show severe events that happen during real distribution.

2. Parcel express companies use others vehicles like minivans, mid-size or heavy trucks to deliver shipments; therefore, data from this study can have limited use for the entire parcel delivery chain, but covers the important portion related to stacked parcels. In a trailer such shipments are unitized in sacks, bundles or pallets and require cargo securement regulations so they are blocked and braced with dunnage or straps and load bars. In addition, parcels travel in heavy truck or mid-size trucks to get to their final destination and operators use bigger containers, such as roll or cage containers, to group the packages together. At this step various packages are grouped together in real life and it is very rare to have identical parcels stacked together.

3. Here has to be noted that the packages during the distribution suffer vibration events in the lateral and longitudinal excitation also, but the widespread practice in the laboratory use single degree-of-freedom (SDOF) vibration system. Thus, the package does receive a more complex input vibration in the real practice, which can cause more complex vibration response between the layers of packages.

4. Last, but not least, the theoretical dynamics of stacked parcels can be described as a non-linear free vibration of coupled mass-spring system, thereby the various geometrical sizes and weights of packages in the stacked unit load will change vibration intensity in a non-linear way. 

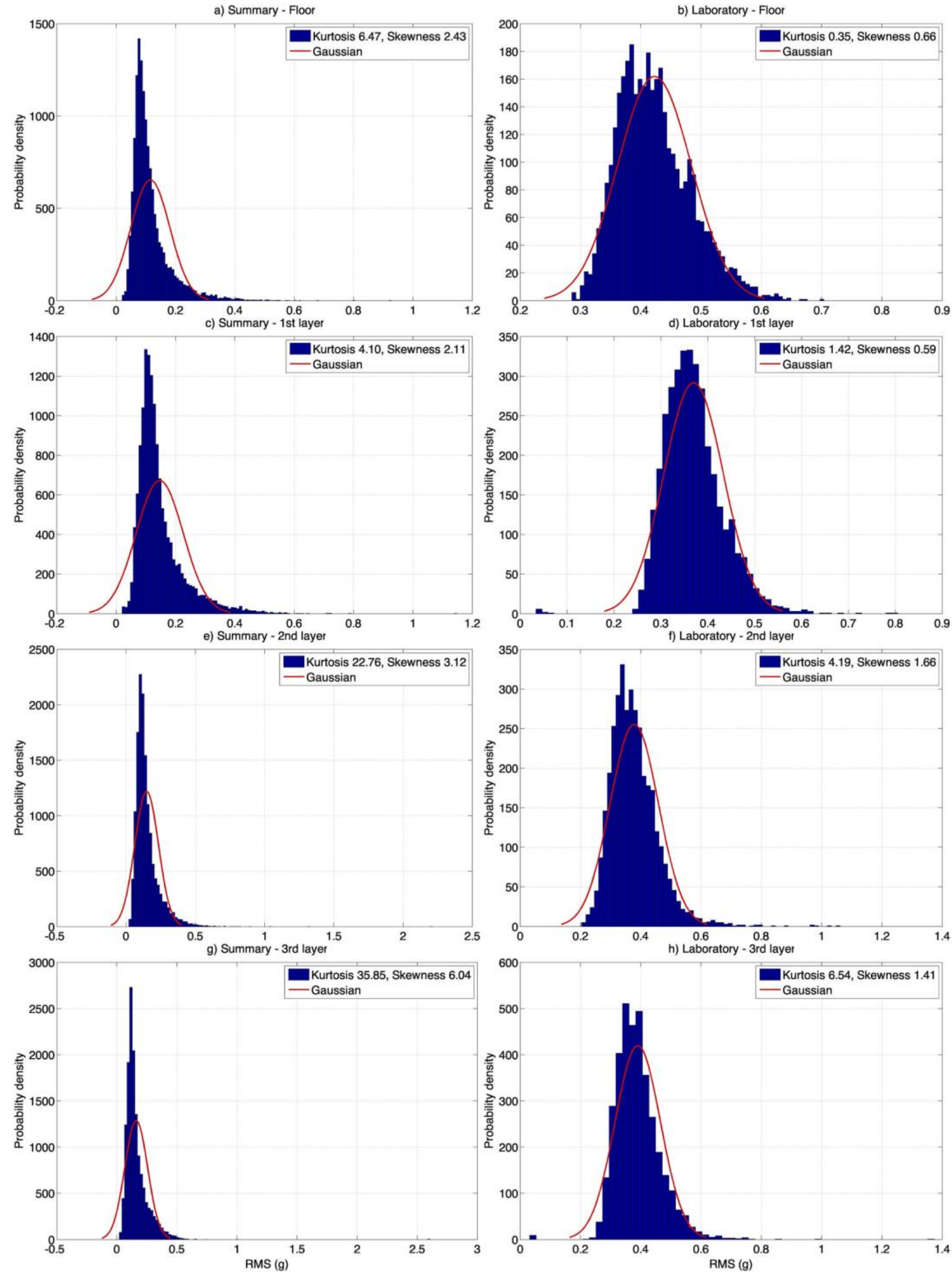

Figure 10. PDF for recorded vertical RMS (g) at field measurement and in laboratory at, (a) Summary-Floor, (b) LaboratoryFloor, (c) Summary-1st layer, (d) Laboratory-1st layer, (e) Summary-2nd layer, (f) Laboratory-2nd layer, (g) Summary-3rd layer, (h) Laboratory-3rd layer. 


\section{Conclusions}

In express stacked parcels delivery without vertical fixation the higher parcel position has a tendency to have higher acceleration peaks and RMS acceleration. Among various road conditions the urban road produced the highest acceleration events. This was $4.63 \mathrm{~g}$ for this study in the 3rd level of a stacked unit. It is clear based on the data that all accelerations above $1 \mathrm{~g}$ cause parcels to be air-lifted.

This increasing phenomenon can also be observed at the vibration intensity, but the field vibration resulted in much lower vibration intensity than the laboratory test $(1-200 \mathrm{~Hz})$ frequency range), even in the top row of the unit where it was observed that the field vibration intensity was the highest. The vibration intensity in the field was higher in any unit level than the vibration intensity on the floor due to the out-of-phase motion during distribution. This not true for the laboratory simulation where the PD levels were always higher on the vibration table in regard to the in-phase motion of the unit.

The PD levels of the layers in the stacked unit in the field, in the frequency range of $7-50 \mathrm{~Hz}$, practically reached or exceeded the levels of the ASTM D7386 test spectrum, so here the artificial amplification of the test spectrum cannot be interpreted. In the $1-6 \mathrm{~Hz}$ lower frequency range and over $50 \mathrm{~Hz}$ the response vibration of the unit rows was significantly lower than the test spectrum. Therefore, the time-compression method of standard protocol can be determined even for the top level of a stacked unit without fixation.

The authors further suggest for the applied distribution practice that it is advisable to place packages on the top row of the set that are less sensitive to vibration on the one hand, or to use damping materials that have more favorable damping properties between $7-50 \mathrm{~Hz}$. Furthermore, in laboratory tests, the time compression methods used by the standards should only be carefully considered in the frequency range of $7-50 \mathrm{~Hz}$ to reproduce random vibrations generated for upper level of stacked packages. Further consideration should be given to securing the stacked sensitive goods to avoid amplification of vibration intensity at the top of the stacked unit.

Author Contributions: P.B. and B.M. designed the research. B.M. conducted measurements and experiments; P.B. and B.M. developed and analyzed the data and presented results. B.M. wrote the original draft; P.B. finalized the draft. This research project was conducted under the supervision of P.B. All authors have read and agreed to the published version of the manuscript.

Funding: This research received no external funding.

Institutional Review Board Statement: Not applicable.

Informed Consent Statement: Not applicable.

Data Availability Statement: The data presented in this study are available on request from the corresponding author.

Conflicts of Interest: The authors declare no conflict of interest. 
Appendix A. Specifications of CDFs for Recorded Acceleration Peaks for This Study

\begin{tabular}{|c|c|c|c|c|c|c|c|}
\hline Data & $\begin{array}{c}\text { Location of } \\
\text { Measurement }\end{array}$ & $\begin{array}{c}\text { Location of } \\
\text { Saver }\end{array}$ & $\begin{array}{l}\text { Acc. at } 50 \% \\
\text { Occ. }\end{array}$ & $\begin{array}{c}\text { Acc. at } 90 \% \\
\text { Occ. }\end{array}$ & $\begin{array}{l}\text { Acc. at } 95 \% \\
\text { Occ. }\end{array}$ & $\begin{array}{c}\text { Acc. at } 99 \% \\
\text { Occ. }\end{array}$ & $\begin{array}{c}\text { Max. } \\
\text { Acceleration }\end{array}$ \\
\hline \multirow{24}{*}{$\begin{array}{l}\text { Acceleration } \\
(\mathrm{g}, \text { peak })\end{array}$} & \multirow{4}{*}{ Motorway } & Floor & 0.28 & 0.46 & 0.55 & 0.92 & 1.77 \\
\hline & & 1st Layer & 0.34 & 0.50 & 0.63 & 2.09 & 2.88 \\
\hline & & 2nd Layer & 0.35 & 0.52 & 0.66 & 2.21 & 2.94 \\
\hline & & 3rd Layer & 0.35 & 0.57 & 0.72 & 1.24 & 3.04 \\
\hline & \multirow{4}{*}{ Arterial road } & Floor & 0.31 & 0.56 & 0.70 & 1.03 & 1.89 \\
\hline & & 1st Layer & 0.40 & 0.71 & 0.86 & 1.34 & 2.73 \\
\hline & & 2nd Layer & 0.43 & 0.80 & 0.98 & 1.52 & 2.63 \\
\hline & & 3rd Layer & 0.44 & 0.88 & 1.09 & 1.56 & 3.98 \\
\hline & \multirow{4}{*}{ Country road } & Floor & 0.29 & 0.80 & 1.02 & 1.55 & 2.40 \\
\hline & & 1st Layer & 0.35 & 0.94 & 1.26 & 1.88 & 3.33 \\
\hline & & 2nd Layer & 0.39 & 0.99 & 1.24 & 1.80 & 3.60 \\
\hline & & 3rd Layer & 0.42 & 1.10 & 1.33 & 1.88 & 3.85 \\
\hline & \multirow{4}{*}{ Urban road } & Floor & 0.34 & 0.84 & 1.05 & 1.66 & 3.00 \\
\hline & & 1st Layer & 0.42 & 1.04 & 1.31 & 1.89 & 3.00 \\
\hline & & 2nd Layer & 0.43 & 1.07 & 1.34 & 1.99 & 3.29 \\
\hline & & 3rd Layer & 0.45 & 1.18 & 1.43 & 2.01 & 4.63 \\
\hline & \multirow{4}{*}{ Summary } & Floor & 0.30 & 0.67 & 0.88 & 1.41 & 2.80 \\
\hline & & 1st Layer & 0.38 & 0.87 & 1.11 & 1.70 & 3.33 \\
\hline & & 2nd Layer & 0.38 & 0.87 & 1.16 & 2.44 & 3.78 \\
\hline & & 3rd Layer & 0.39 & 0.98 & 1.25 & 2.11 & 4.63 \\
\hline & \multirow{4}{*}{ Laboratory } & Floor & 1.49 & 1.84 & 1.94 & 2.18 & 2.86 \\
\hline & & 1st Layer & 0.99 & 1.25 & 1.38 & 1.83 & 3.96 \\
\hline & & 2nd Layer & 1.03 & 1.37 & 1.50 & 2.68 & 4.72 \\
\hline & & 3rd Layer & 1.06 & 1.57 & 1.84 & 2.68 & 6.53 \\
\hline
\end{tabular}

\section{Appendix B. Specifications of CDFs for Recorded RMS Acceleration Values for This Study}

\begin{tabular}{|c|c|c|c|c|c|c|c|}
\hline Data & $\begin{array}{c}\text { Location of } \\
\text { Measurement }\end{array}$ & $\begin{array}{c}\text { Location of } \\
\text { Saver }\end{array}$ & $\begin{array}{c}\text { RMS at } 50 \% \\
\text { Occ. }\end{array}$ & $\begin{array}{c}\text { RMS at } 90 \% \\
\text { Occ. }\end{array}$ & $\begin{array}{c}\text { RMS at } 95 \% \\
\text { Occ. }\end{array}$ & $\begin{array}{c}\text { RMS at } 99 \% \\
\text { Occ. }\end{array}$ & $\begin{array}{c}\text { Max. } \\
\text { Acceleration }\end{array}$ \\
\hline \multirow{24}{*}{ RMS (g) } & \multirow{4}{*}{ Motorway } & Floor & 0.09 & 0.13 & 0.15 & 0.22 & 0.39 \\
\hline & & 1st Layer & 0.11 & 0.15 & 0.17 & 0.25 & 0.51 \\
\hline & & 2nd Layer & 0.12 & 0.16 & 0.18 & 0.25 & 0.55 \\
\hline & & 3rd Layer & 0.12 & 0.18 & 0.20 & 0.28 & 0.61 \\
\hline & \multirow{4}{*}{ Arterial road } & Floor & 0.10 & 0.16 & 0.20 & 0.28 & 0.46 \\
\hline & & 1st Layer & 0.13 & 0.22 & 0.26 & 0.35 & 0.67 \\
\hline & & 2nd Layer & 0.14 & 0.22 & 0.27 & 0.39 & 0.71 \\
\hline & & 3rd Layer & 0.15 & 0.25 & 0.30 & 0.39 & 0.71 \\
\hline & \multirow{4}{*}{ Country road } & Floor & 0.09 & 0.25 & 0.30 & 0.42 & 0.68 \\
\hline & & 1st Layer & 0.12 & 0.30 & 0.35 & 0.49 & 0.78 \\
\hline & & 2nd Layer & 0.13 & 0.31 & 0.38 & 0.49 & 0.89 \\
\hline & & 3rd Layer & 0.14 & 0.33 & 0.38 & 0.51 & 1.01 \\
\hline & \multirow{4}{*}{ Urban road } & Floor & 0.11 & 0.23 & 0.29 & 0.41 & 0.80 \\
\hline & & 1st Layer & 0.13 & 0.30 & 0.36 & 0.47 & 0.93 \\
\hline & & 2nd Layer & 0.14 & 0.30 & 0.37 & 0.51 & 1.15 \\
\hline & & 3rd Layer & 0.15 & 0.33 & 0.39 & 0.49 & 1.32 \\
\hline & \multirow{4}{*}{ Summary } & Floor & 0.10 & 0.20 & 0.25 & 0.37 & 0.93 \\
\hline & & 1st Layer & 0.12 & 0.25 & 0.31 & 0.44 & 1.15 \\
\hline & & 2nd Layer & 0.13 & 0.25 & 0.32 & 0.45 & 2.21 \\
\hline & & 3rd Layer & 0.13 & 0.28 & 0.34 & 0.46 & 2.61 \\
\hline & \multirow{4}{*}{ Laboratory } & Floor & 0.42 & 0.51 & 0.53 & 0.59 & 0.70 \\
\hline & & 1st Layer & 0.36 & 0.45 & 0.48 & 0.55 & 0.81 \\
\hline & & 2nd Layer & 0.37 & 0.47 & 0.51 & 0.64 & 1.06 \\
\hline & & 3rd Layer & 0.38 & 0.49 & 0.52 & 0.61 & 1.37 \\
\hline
\end{tabular}


Appendix C. Kurtosis and Skewness Values for the Various Measurements for This Study

\begin{tabular}{|c|c|c|c|c|c|}
\hline $\begin{array}{c}\text { Road } \\
\text { Conditions }\end{array}$ & Location & $\begin{array}{c}\text { Kurtosis } \\
\text { (Acceleration } \\
\text { Data, g Peak) }\end{array}$ & $\begin{array}{c}\text { Skewness } \\
\text { (Acceleration } \\
\text { Data, g Peak) }\end{array}$ & Kurtosis (RMS g Data) & Skewness (RMS g Data) \\
\hline \multirow{4}{*}{ Motorway } & Floor & 1.85 & -1.15 & 11.64 & 2.83 \\
\hline & 1st & 1.75 & 1.23 & 10.35 & 2.42 \\
\hline & 2nd & 3.71 & 1.89 & 6.29 & 2.15 \\
\hline & 3nd & 7.27 & 0.29 & 16.16 & 2.63 \\
\hline \multirow{4}{*}{ Arterial road } & Floor & 1.89 & -0.98 & 3.18 & 1.93 \\
\hline & 1st & 1.21 & 0.42 & 3.54 & 1.96 \\
\hline & 2nd & 0.03 & 0.59 & 2.47 & 1.68 \\
\hline & 3nd & 0.59 & 0.74 & 1.82 & 1.44 \\
\hline \multirow{4}{*}{ Summary } & Floor & 1.67 & -0.62 & 6.47 & 2.43 \\
\hline & 1st & 0.51 & 0.11 & 4.10 & 2.11 \\
\hline & 2nd & 1.04 & 1.51 & 22.76 & 3.12 \\
\hline & 3nd & -0.33 & 1.03 & 35.85 & 6.04 \\
\hline \multirow{4}{*}{ Country road } & Floor & 1.89 & -0.55 & 0.57 & 1.72 \\
\hline & 1st & -0.90 & 0.22 & 1.54 & 1.49 \\
\hline & 2 nd & -1.25 & 1.05 & 1.66 & 1.69 \\
\hline & 3nd & -2.83 & 0.43 & -0.25 & 1.24 \\
\hline \multirow{4}{*}{ Urban road } & Floor & 0.07 & -0.27 & 2.31 & 1.88 \\
\hline & $1 \mathrm{st}$ & -1.83 & 0.25 & 1.55 & 1.62 \\
\hline & 2nd & -2.25 & 0.70 & 2.23 & 1.72 \\
\hline & 3nd & -2.52 & 0.46 & 2.73 & 1.59 \\
\hline \multirow{4}{*}{ Laboratory } & Floor & -0.17 & -0.85 & 0.35 & 0.66 \\
\hline & 1st & 1.30 & -1.30 & 1.42 & 0.59 \\
\hline & 2nd & 1.82 & 0.61 & 4.19 & 1.66 \\
\hline & 3nd & 1.49 & 3.50 & 6.54 & 1.41 \\
\hline
\end{tabular}

\section{References}

1. Mazarenau, E. Global Size of the Courier, Express and Parcel (CEP) Market 2009-2019. Statista.com. Available online: https: //www.statista.com/statistics/723986/cep-market-total-revenue-worldwide/ (accessed on 14 November 2020).

2. Böröcz, P.; Molnár, B. Measurement and Analysis of Vibration Levels in Stacked Small Package Shipments in Delivery Vans as a Function of Free Movement Space. Appl. Sci. 2020, 10, 7821. [CrossRef]

3. Brandenburg, R.K.; Lee, J.J. Fundamentals of Packaging Dynamics; L.A.B. Equipment, Inc.: Itasca, IL, USA, 2001.

4. Böröcz, P.; Singh, S.P. Measurement and analysis of delivery van vibration levels to simulate package testing for parcel delivery in Hungary. Packag. Technol. Sci. 2018, 31, 342-352. [CrossRef]

5. Zhou, H.; Wang, Z.W. Measurement and analysis of vibration levels for express logistics transportation in South China. Packag. Technol. Sci. 2018, 31, 665-678. [CrossRef]

6. Chonhenchob, V.; Singh, S.P.; Singh, J.; Stallings, J.; Grewal, G. Measurement and analysis of vehicle vibration for delivering packages in small-sized and medium-sized trucks and automobiles. Packag. Technol. Sci. 2012, 25, 31-38. [CrossRef]

7. Stallings, J.; Singh, J.; Singh, S.P. Measurement and Analysis of Vehicle Vibration for Parcel Delivery Vehicles in Single Parcel Shipments. J. Appl. Packag. Res. 2010, 4, 75-82.

8. Park, J.; Choi, S.; Jung, H.M. Measurement and Analysis of Vibration Levels for Truck Transport Environment in Korea. Appl. Sci. 2020, 10, 6754. [CrossRef]

9. Zhou, H.; Wang, Z.W. Comparison study on simulation effect of improved simulation methods for packaging random vibration test. Packag. Technol. Sci. 2019, 32, 119-131. [CrossRef]

10. Böröcz, P. Vibration levels in vans as a function of payload and leaf spring sheet number. J. Test. Eval. 2017, 46, 236-243. [CrossRef]

11. ASTM D7386-16. Standard Practice for Performance Testing of Packages for Single Parcel Delivery Systems; ASTM International: West Conshohocken, PA, USA, 2016. Available online: www.astm.org (accessed on 15 January 2021).

12. Garcia-Romeu-Martinez, M.-A.; Singh, S.P.; Cloquell-Ballester, V.-A. Measurement and analysis of vibration levels for truck transport in Spain as a function of payload, suspension and speed. Packag. Technol. Sci. 2008, 21, 439-451. [CrossRef]

13. Lu, F.; Ishikawa, Y.; Kitazawa, H.; Satake, T. Effect of vehicle speed on shock and vibration levels in truck transport. Packag. Technol. Sci. 2010, 23, 101-109. [CrossRef]

14. Rouillard, V.; Sek, M.A. Synthesizing nonstationary, non-Gaussian random vibrations. Packag. Technol. Sci. 2010, 23, 423-439. [CrossRef]

15. Garcia-Romeu-Martinez, M.-A.; Rouillard, V. On the Statistical Distribution of Road Vehicle Vibrations. Packag. Technol. Sci. 2011, 24, 451-467. [CrossRef]

16. Shires, D. On the time compression (test acceleration) of broadband random vibration tests. Packag. Technol. Sci. 2011, 24, 75-87. [CrossRef] 
17. Kipp, W.I. Vibration Testing Equivalence: How Many Hours of Testing Equals How Many Miles of Transport? International Safe Transit Association: East Lansing, MI. Paper Originally Presented at ISTA Conference 2000 Updated December 2008. Available online: http:/ / www.wikippco.com/Vib\%20Test\%20Equivalence.pdf (accessed on 14 November 2020).

18. Rouillard, V. On the Non-Gaussian Nature of Random Vehicle Vibrations. In Proceedings of the World Congress on Engineering, London, UK, 2-4 July 2007; Volume II.

19. Otari, S.; Odof, S.; Nolot, J.B.; Vasseur, P.; Pellot, J.; Krajka, N.; Erre, D. Statistical characterization of acceleration levels of random vibrations during transport. Packag. Technol. Sci. 2011, 24, 177-188. [CrossRef]

20. Garcia-Romeu-Martinez, M.A.; Rouillard, V.; Cloquell-Ballester, V.A. A Model for the Statistical Distribution of Road Vehicle Vibrations. In Proceedings of the World Congress on Engineering, London, UK, 2-4 July 2007; Volume II.

21. Böröcz, P. Averaged vibration levels during courier parcel delivery service in small truck in Hungary. FME Trans. 2018, 46, 211-217. [CrossRef] 